\title{
Drawing Insights from Cognitive Science for a Strengthened Teacher Preparation: Learnings from Optics
}

\author{
Gurjeet Kaur \\ Department of Teacher Training \& Non-Formal Education, Institute of Advanced Studies in \\ Education, Faculty of Education Jamia Millia Islamia, India
}

\begin{abstract}
This paper discusses the effectiveness of an intervention in optics that was developed to bridge the gap between the 'shared' and the alternative conceptions of future physics teachers. This paper describes the process beginning from the identification of the learning needs of the target group to the development of the instructional modules to the analysis of the data. To ascertain the effectiveness of the developed material in facilitating conceptual change the data were examined at three levels - prior to, during the course of and post intervention. The responses of students on one item are discussed in detail. In addition to studying the ideas before and after instruction which is the norm in such type of studies it was also thought important to study closely the thought processes of the students during the intervention. The learning derived from this endeavor is then reflected upon. The implications of the study for strengthening the teacher preparation process are discussed.
\end{abstract}

\section{Introduction}

Different perspectives in cognitive studies have propounded a different view of nature of learning. While the behavioral view emphasizes that learning can be understood in terms of overt behavior and the environmental factors influencing it, cognitive psychologists hold that certain processes in the learners' brain mediate learning and social psychologists propound that learning is embedded in one's socio-cultural context. We perceive that as our understanding about the learner and the processes of education has moved ahead, the 'impersonal' and 'objective' view of learning has gradually given way to a 'personal' and a 'subjective' viewpoint.

In the emergent constructivist paradigm, learning is viewed as 'construction' (rather than acquisition) and assimilation (instead of adoption) of knowledge using the cognitive tools available to an individual. The personal construction of knowledge by an individual learner is understood to be influenced by her/his prior knowledge, experience and the social context in which learning takes place. A familiarity with the content and structure of learners' knowledge is therefore essential for the constructivist understanding of science learning and for the designing of science curricula and teaching tools. Learners' ideas of the world have therefore been the subjects of extensive investigation in last three decades. Learners' ideas in physics have been specifically researched extensively probably because many of the concepts of physics are particularly counter-intuitive.

\section{Understanding Learners’ Ideas}

Our growing understanding about the teaching learning processes has increasingly shifted the spotlight from the teacher onto the learner. Learner is no longer viewed as the traditional 'blank slate' but instead as an active 'constructor' (rather than acquirer) and 'assimilator' (instead of adopter) of knowledge. This viewpoint has encouraged us to make efforts to get to know the learner better. Research in the areas of Cognitive Psychology and Science Education over the last three decades has demonstrated that children come to formal science instruction with their own views and explanations of the world, natural phenomena and surrounding events. They may not even always be consciously aware of these explanations. These explanations are invariably based on their everyday experiences and constitute what has been called the 'commonsense reasoning' by Bliss [2] and 'naïve physics' by Reiner, Slotta, Chi and Resnick [17]. This intuitive knowledge was called 'children's science' by Gilbert, Osborne and Fensham and highlighted the fact that children begin to develop their personal world views very early [8]. These personal world views then influence future learning by forming a conceptual backdrop against which future experiences and instruction are interpreted. An interesting aspect of these ideas from the teacher education perspective is that many of these ideas are tenacious and may continue to exist despite several years of formal science instruction and in case of teachers, if left unaddressed these could then be passed on to children. Mintzes and Chiu also point out that learners often hold a host of alternative scientific and mathematical views which contrast with those offered by scientists, teachers and textbooks and many of these alternative views are found in males and females of all ages, abilities, social classes and cultures, though other views may be unique and culture bound [11]. The origin of the 
term 'alternative conceptions' may be attributed to the term 'alternative frameworks' which was originally used by Driver to describe certain ideas that children come to school with which do not coincide with the scientific view [3]. The term 'alternative conceptions' when initially used in the Education literature referred primarily to preconceptions, naïve ideas or intuitive notions (i.e. conceptions held before receiving formal instruction) of children. However, gradually the scope of the term expanded to include all ideas that varied from the currently accepted scientific understandings. Traditionally, all ideas that were different from the scientific view were called the 'misconceptions' because they were considered to be incorrect and inaccurate conceptions that obstructed learning but the constructivist literature looks at these ideas differently. These ideas, instead of being considered as obstructions to be overcome are rather taken as starting points for future instruction. Further, many times these ideas may not be entirely incorrect but may incorporate certain correct features as well. Some of these ideas may also be pre conceptions that have been resistant to formal instruction. The learners may also at times evolve some kind of 'hybrid knowledge' which incorporates ideas from classroom instruction as well as some beliefs students held prior to the instruction [5]. Gilbert described alternative conceptions as ideas which differ significantly from the accepted scientific view at any time [7]. Pardhan and Bano who examined science teachers' alternate conceptions about direct current say that alternative ideas are those scientific ideas held by individuals which do not match expert scientific views [16]. They elaborate that though these ideas have been given different names but the most appropriate is 'alternative conceptions' because these ideas are individual's mental constructs, which make sense to the individual and work for the individual to make sense of new knowledge.

The term 'alternative conceptions' may therefore be understood to include within its purview:

- Pre-conceptions that have survived formal instruction.

- 'Hybrid conceptions resulting from the interplay between formal and pre conceptions. These may not be entirely incorrect ideas but may incorporate some correct ideas as well.

- $\quad$ Limited conceptions.

\section{Teacher Preparation, Subject Matter Knowledge and Conceptual Change}

Many studies have pointed out that school teachers may often subscribe to the same alternative conceptions as the learners [1],[11] and [16]. That the alternative ideas may often resist and defy formal instruction has also been vehemently stated [12].
Nussbaum attributes this to the tendency towards 'subject matter approach' to the task of concept analysis as opposed to the 'cognitive approach' [15]. In a subject matter approach the important relevant and advanced ideas that represent the mature state of the discipline are extracted from the text book whereas the preliminary ideas are taken for granted. This could pose problems when the preliminary ideas themselves are primitive and at variance with the scientifically accepted view.

Traditionally, teachers acquired disciplinary knowledge in the respective university departments and pedagogical knowledge in the departments of Education. The disciplinary knowledge and the pedagogical knowledge were viewed as different knowledge domains. The need to combine the two is now being recognized and this integrated knowledge domain has been termed as 'Pedagogical Content Knowledge' in a few studies. Etkina described it as an application of general subject independent knowledge of how people learn to the knowledge of particular subject matter [4]. Teacher Education programmes have only recently begun to acknowledge the gaps in, and address the need for enhancing future teachers' conceptual understanding in different disciplines. Mestre who made some pointed research based suggestions about physics courses for prospective science teachers also stressed that teaching of content should be the central focus and that emphasis should be on in depth understanding wherein qualitative reasoning based on physics concepts is encouraged [13]. 'National Curriculum Framework For Teacher Education'(NCFTE) which is the most recent guiding document for teacher education in India, specifically talks about the importance of engaging the future teachers with the conceptual knowledge that they have gained through general education[14]. NCFTE notices that most teacher education courses do not focus on subject content and the prior knowledge of the teacher trainees is taken for granted which can be drawn upon whenever required but to enable the trainees to present the subject content in developmentally appropriate ways, they will have to be made to revisit and reconstruct various theoretical concepts.

\section{Choosing the Area of Investigation}

\subsection{Examining the Research Pointers}

A substantive amount of literature in science education points to significant gaps in understanding of pre service and in-service teachers in different areas. 'Light' has been widely reported as an area prone to particularly resistant alternative conceptions or as Reiner et al. call them 'robust misconceptions' [17]. Galili and Hazen cite many reasons for this which are: 
- The physical parameters associated with light, e.g. its speed, wavelength, pressure and discrete nature, are all far removed from the range of perception of the human senses.

- The optical phenomena are commonly observed in media (air, water) which often greatly modify the behavior of light from that in vacuum.

- Many linguistic constructions do not conform to present-day scientific knowledge. Phrases such as 'her eyes shine', ‘his face radiates light', 'she casts a glance' may cause confusion.

- Full comprehension in Optics requires insights from different disciplines. Physics (the nature of light), physiology (the functioning of the eye) and psychology (the interpretation of visual and color perception) are all needed for comprehensive discussions of optical phenomena.

- Optics instruction is heavily based on graphic symbolism which may not be easy to interpret [6].

In addition to the above, study of optics relies on different explanatory frameworks (such as the ray optics, wave optics and the quantum optics) that are all correct in certain contexts but students encounter them at different levels of their academic lives. There is little scope for students to be able to juxtapose these frameworks and view them in relation to each other. All these factors then cumulatively make optics a highly counter-intuitive area of study and a fertile ground for alternative conception research.

\subsection{Gauging the ideas of in service teachers in Optics}

To take an initial look at the prevalence of alternative ideas among in-service teachers, a tool consisting of 18 statements was administered to 25 in-service teachers. Of these 3 statements were correct and 15 were incorrect. The correct statements were: a) Size of lens does not determine the size of image and b) Light can cancel light. c) How far light would extend outwards from a source is independent of the brightness/intensity of the source. The incorrect statements included such statements as :a) Photons are particles which are called light wave when they move together. b) Only two light rays one from the top and the other from the bottom of an object form the image of the object. The incorrect statements reflected several 'alternative ideas' in optics that the research studies had highlighted. The statements were to be marked as 'true' or 'false'. The teachers were a mixed group who taught science at middle, secondary and senior-secondary level. Being fully aware of the limitations of a T-F format, the instrument was only used as a rough gauge. The examination of the teachers' responses gave the following graph. The 18 ideas are shown on the Xaxis and the no. of teachers on the $\mathrm{Y}$-axis

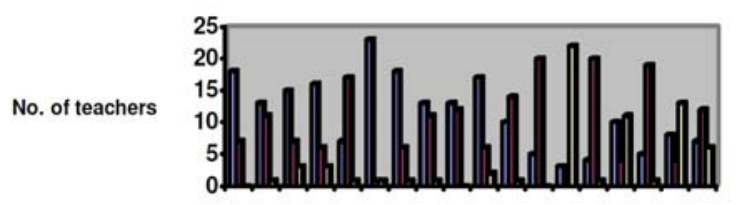

口correct responses $\square$ incorrect responses $\square$ no response

Figure 1. Responses of the in service teachers on the $\mathrm{T} / \mathrm{F}$ instrument

Even though the sample was small and the results may at best be considered illustrative, they do call for a deeper probe of the teachers' ideas and the need to bring out a systemic reform by examining and addressing the ideas of pre service teachers is indicated.

\section{The Study}

\subsection{Groundwork}

The area chosen for investigation was 'optics'. Various alternative ideas in optics as documented in the related studies were identified. However, as alternative ideas do not, in general, seem to respect age or educational qualifications; it was difficult to say if these ideas were likely to be held by preservice teachers. To ascertain the possible prevalence of these ideas among pre-service teachers, a list of possible alternative ideas was prepared and three teacher educators involved in teaching physics to future teachers were asked to give their opinion on a three point scale regarding the likeliness of these ideas of being found among future teachers. Simultaneously, a document highlighting the 'shared conception' for each 'alternative conception' was prepared and ratified through discussion with 2 physicists.

\subsection{Sequential Steps}

Step I: The study was initiated by putting some questions to a group of 13 students enrolled at an institution in a one year degree programme in Education. The interviews were semi-structured but were guided by the following questions: What is light? Do we see light? How do we see things? How are images formed in mirrors?

Step II: Based on the pointers given by the interviews, a series of 4 one hour interactions were planned with the same group to further gauge their ideas. The interactions revolved around very basic ideas about vision, sight, image formation in mirrors and lenses and colors. 
Step III: The interactions with the learners were examined to look for the presence of alternative conceptions. Relevant research studies were scanned to search for appropriate probes that could help detect these conceptions. Some of these probes were then converted into multiple choice type items by introducing suitable distracters on the basis of the ideas pointed to by these studies and on the basis of the learners' responses during the interactions with them. For certain ideas for which no suitable questions were found, the questions were selfdesigned.

Step IV: The final tool consisted of 16 multiple choice type and 7 free response items. These questions were then administered in the next session on a group of 17 students enrolled in the same program in the same institution and on 10 students enrolled in the same program in another institution. Certain changes were made on the basis of whether or not these could be comprehended by the future teachers.

Step V: The responses on these set of questions were analyzed and the discrepancies between the alternative and shared conceptions were reflected upon. 5 modules that constituted the intervention referred to in this study were developed. These modules attempted to address the 'alternative conceptions of the learners not directly but obliquely by making them rethink, revisit and reconstruct the subject matter in optics as it developed historically and as it came to be represented in the text books.

Step VI: A post-test paralleling the pre-test was prepared and validated through expert opinion.

Step VII: The modules were then tried out with a group of 14 students and modified in the light of the try out.

Step VIII: A group of 72 pre-service teachers (future teachers) across 3 teacher education institutions formed the sample of the study and apre assessment was carried out. The sampling was purposive and those institutions were included that agreed to provide the researcher with the time and space to carry out long drawn interactions with the future teachers. Though the students differed in their educational qualifications, some being graduates and other post graduates, they all had studied physics at the undergraduate level and all of them had opted for physics as one of their teaching subjects.

Step IX: The developed modules were transacted with this group and subsequently, a post assessment session was held. Of these 72 participants, there were 56 who worked on all the 5 modules. The performance of these future teachers during the preand post-interventional assessment and their responses on the modules constituted the data.

Step X: Students' performance was analyzed and findings were reported.

\section{Pre Interventional Assessment}

The time for carrying out pre assessment was one and a half to two hours. The tool was translated for the students who reported a difficulty in comprehension. The instructions clearly stated that the assessment was being carried out purely for research purposes and was in no way meant to influence their grades. Some students who showed a reluctance to write their names were told to write a code instead on the pre assessment sheet as well as on their modules. The students of different institutions were assigned different alphabet series of student codes.

\section{The Intervention}

Before beginning to develop the design, it was important to consider the following core questions:

- How are the alternative conceptions different from the shared conceptions?

- How could the alternative conceptions possibly have been formed?

- How could student teachers be made to look critically at their own conceptions?

- What kind of engagement with subject matter could encourage the student teachers to revise their conceptual understandings? Closely considering the nature of alternative conceptions and reflecting upon their possible causes led to some important observations:

- Many alternative conceptions result from an inappropriate assimilation of formal instruction with prior notions and the represented form of knowledge in Optics may be at the root of several alternative conceptions. Teachers need to be facilitated to appreciate the relationship between the represented world and the real world.

- The role of the self is extremely important in understanding the optical phenomena as the self forms an active part of the optical phenomena and all received information is influenced by the observer. There is a need to actively highlight the role of observer.

- Our understandings get continuously revised as we come across newer evidences that cannot be explained on the basis of present explanations. Tracing the history of a phenomenon can lend us useful insights into why we think about it the way we do.

On the basis of the above considerations and on the basis of the observation that the recent research literature emphasizes the importance of addressing the epistemological assumptions of the learners to improve their conceptual understanding, 5 interventional modules were developed. The modules were titled as follows. 


\subsection{Self, sight and vision}

One interesting reason for light being a curious phenomenon that is difficult to intellectually access is the role of the self and therefore the inherent subjectivity in our perception. This subjectivity cannot be got rid of and operates subconsciously in all our visual interpretations. The problem arises when learners fail to take into account the role of the self and the eye in the process of sight and vision. In this module an attempt is made to assign an active role to the eye in the process of vision by consistently attracting the attention of the future teachers to its presence.

\subsection{Images in Optics}

Images such as diagrams, sketches or any other form of visual representations are an important form of communication in Physics or in any subject for that matter and they have an immense power to shape our understandings of the different aspects of the world. This becomes specifically true for an area such as 'Optics' in which representations such as pictures and diagrams are introduced quite early in the study of the topic. These images begin to act as a crucial link between reality and our conception of it but one must always be alive to the dichotomy between the concrete appearances and the realm of knowledge. Images are after all symbols and it is important that we begin to interpret these symbols in the correct or the intended sense

\subsection{Words and Meanings}

The socio-linguistic perspective in education has led to a wide recognition of the role of language in learning science. The last thirty years have seen a major change in the perceived role of language in knowledge building. Traditionally language was seen as a uni-directional process that proceeded from the speaker to the listener or from text to the reader but the advent of applied cognitive science has ascribed a very active role to the language in the process of construction of knowledge. Language as a symbol system can either facilitate or hinder the conceptual learning in science because the meaning of the words is gauged, inferred or constructed rather than passively received through linguistic representations. Lemke goes so far as to say that the mastery of academic subjects is in fact the mastery of the specialized pattern of language use in those subjects [10]. 'Words and Meanings' makes an attempt to encourage the future teachers to reflect upon the language of 'optics' in such ways as attracting their attention to terms like 'electrons' that reinforce the particle nature of light and obscure the duality or to terms like 'scattering' and 'reflection' that convey similar sense in everyday usage. The future teachers by becoming alive to these inconsistencies are more likely to remain sensitive to them and take them into account when they begin to function as mainstream teachers in future.

\subsection{Building Understandings I: Multiple viewpoints}

Science educationists are now increasingly acknowledging that science learning frequently requires the learners to link and interpret multiple modes of knowledge representation. The term 'multiple modes' could simply refer to the practice of representing a concept through different forms such as the verbal, graphic or numeric [18] or in some cases it could refer to re-embedding the knowledge in different frameworks. Understanding in the area of optics requires 'multiple modes' to be interpreted in both these senses. An interesting feature about the optical knowledge is its historical evolution and the transition from ray to wave to electromagnetic to quantum mechanical optics. It is envisaged that using the history of optics to juxtapose the multiple explanations of the optical phenomena as they have evolved can help the future teachers' access knowledge from different viewpoints. This section begins by highlighting the earliest explanations of the phenomenon of sight and vision such as the 'active vision' theory. The growth of understanding is then traced from Euclidean rays to Newtonian corpuscles to Huygens's constructions to Maxwell's equations and finally to the phenomena of uncertainty and complementarily.

\subsection{Building Understandings II: Exploring the nature of reality}

The purpose of this module was to get the future teachers to appreciate the inherent uncertainty in nature. An attempt is made to wean them away from classical thinking by referring to the experimental observations that are inconsistent with the classical world view. The students are first asked to predict what would happen if a stream of classical particles hit a wall with two small holes and a screen capable of recording the position at which the particles strike. They are then told to reflect upon the implications of obtaining a maxima minima interference pattern with electrons. The inconsistency with the expected classical results is stressed. All evidence points to the fact that if there are multiple paths available between two points A and B, then the probability amplitudes of all these paths must be added to obtain the total amplitude of the process. The curious nature of light is highlighted by citing the 'delayed choice' experiments where the choice of making alternative paths available to light is delayed till the very end long after the light (or the photons) have left the source and yet the results show that all possible 
contributions to the process must be considered for the final pattern and there will only be one final pattern. The notion of uncertainty being a limitation of the measuring instrument is brought into question by citing Bohr's and Heisenberg's Copenhagen interpretation. It is argued that only that explanation of a given phenomenon can be considered viable which successfully interprets and predicts the experimental observations and only the probabilistic interpretation of quantum mechanics fulfils this criterion.

The objective of the modules on 'Building Understandings' is to expose the students to the ways in which understandings in an area evolve over a period of time and circumstances or evidences that lead to old ideas being modified or old explanations being refuted.

\section{Transacting the modules}

The modules were transacted in a specific sequence. It was thought fit to begin the discussion on the nature of knowledge in optics by first considering the role of the self itself in perception. Then the two most fundamental ways of representing knowledge in optics were taken up namely the images and the language. Having thus elaborated upon the different aspects of the represented knowledge in optics, the emergence of the present understandings was historically traced so as to provide the students with a broader perspective on how specific disciplinary knowledge comes about and gets represented in the texts. The most basic ideas in quantum optics, that are extremely counter intuitive such as the uncertainty and complementarity formed the central idea of the last module. This order was kept the same for all the three groups and the total instruction time was 10-12 hours spread over a period of not more than two weeks. The language was English and Hindi translation was provided to those who asked for it. One module was transacted at a time. Students were instructed to follow the format of the material as presented and keep on recording their thoughts, opinions and responses in the space provided as they proceeded.

\section{Post interventional assessment}

The post assessment was carried out in the next session after the students had finished working on the last module. The time duration was about one and a half hour.

\section{Response Analysis}

Responses elicited during the course of pre interventional assessment, transaction of the modules and post interventional assessment were duly recorded and tabulated.

\subsection{Pre- interventional assessment}

Section A (multiple choice type): Each item of the relevant assessment tool was individually taken up. For each item, a table was drawn that mentioned the item no., the given options, the correct option, the alternative conception reflected in each of the distracters, the corresponding shared conception(s), codes of students who chose each option and the total no. of students choosing a particular option. Section B(free response type) : For each item, the specific features of the responses given by the participants were examined. Key features of the responses were culled out. For all the features, corresponding shared/alternative conception was identified. For each item, a table was drawn that depicted the item no., the features of the response, the corresponding shared/alternative conception and codes of students who gave a particular kind of responses.

A question used during pre assessment: The figure below shows an object in front of a plane mirror. The object is in the form of two cubes linked together. One cube is white and the other is painted red It is observed by three observers at positions A, $\mathrm{B}$ and $\mathrm{C}$.

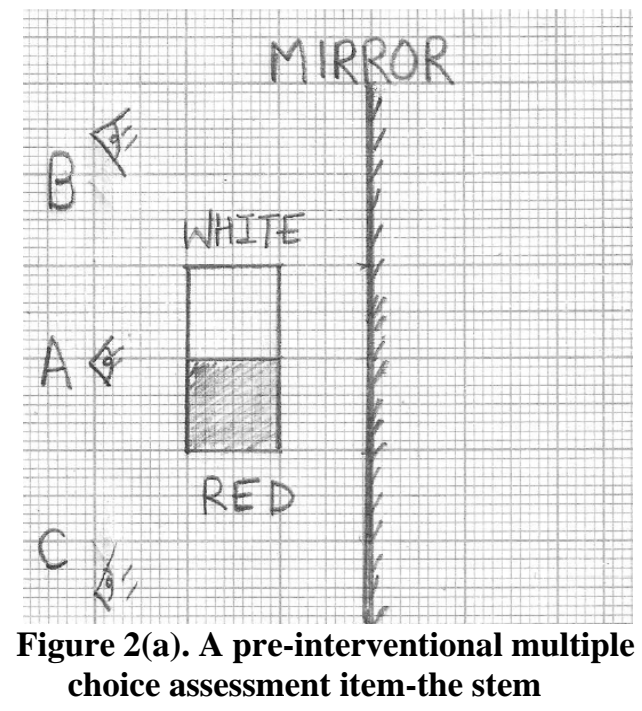

Which among the following diagrams best depicts the relative size, position and the distance of the image for the three observers? 

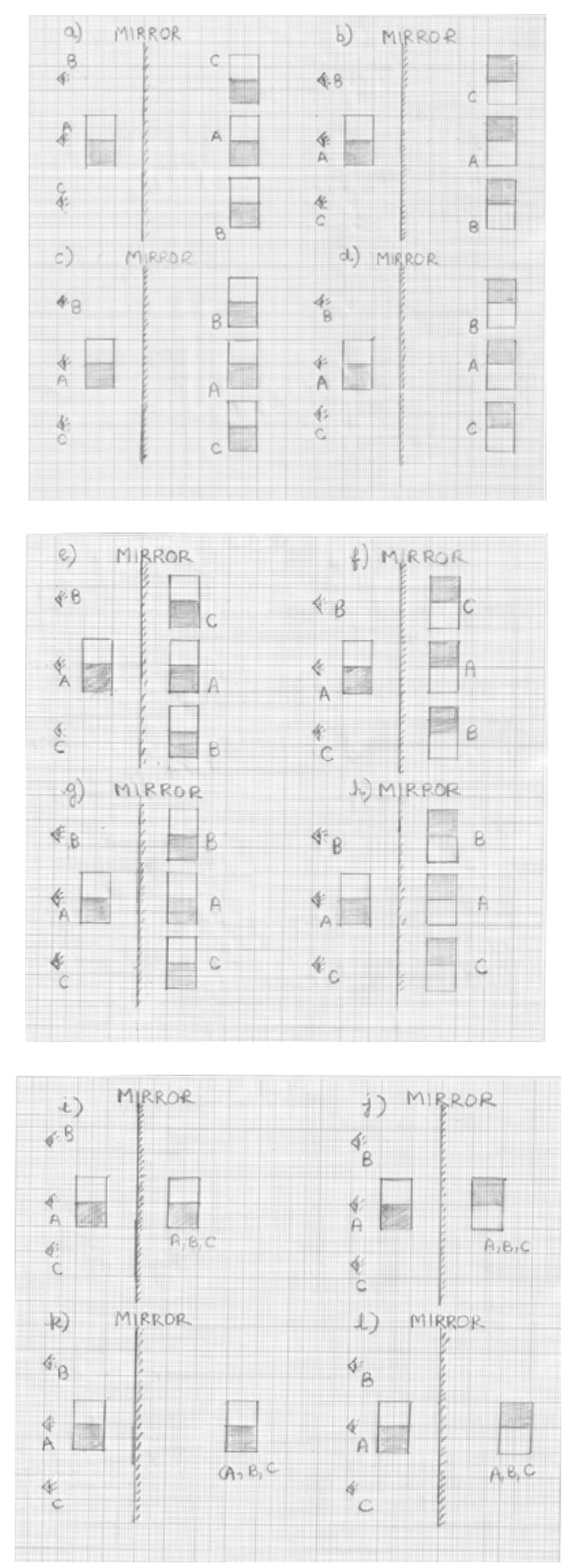

Figure (2b). Options provided

This item has been adapted from the initial version used by Heywood [9].

This item tries to investigate the prevalence of three conceptions: In case of an object placed in front of a mirror- a) The distance of the image from the mirror is determined by the distance of the observer from the mirror. b) The position of the image of an object in a plane mirror is determined by the position of the observer. c) The rays forming the image of an object travel holistically to the mirror and get laterally inverted. The different alternative responses documented in the research studies and those that students gave during the interactions with them were embedded in the different distracters. So if for instance, a participant picked option d), this indicated that this person subscribed to all the three alternative conceptions being investigated while if she/he picked option k), then that was indicative of one out of these three alternative conceptions namely that the image distance of an object placed in front of a plane mirror is determined by the observer distance. Option i) which showed- the image distance as equal to the object distance, the same image for all the three observers and orientation of the image similar to that of the object was indicative of the 'shared conception.' All options were therefore a window to the future teachers' underpinning ideas about light.

\subsection{Tabulating the future teachers' responses on the modules}

There were 5 modules and each module addressed a specific aspect of the nature of knowledge in optics. The writing style was interactive and the participants were expected to respond to certain questions as they proceeded with their work. This enabled a close study of their thought process as they progressed. For each module, queries posed were tabulated. The key features of the responses received to each of the query were identified. On the basis of whether or not these responses resonated with the primary objective of that module, they were categorized as positive, negative or neutral. Given below is an excerpt from the module 'Images in Optics':

Shown below are three ways in which a sun as been depicted. One is a drawing made by a child, one is a picture taken from a rhyme book meant for children at pre primary level and the third has been taken from a story book meant for young children . Look closely at the way source of light is drawn.

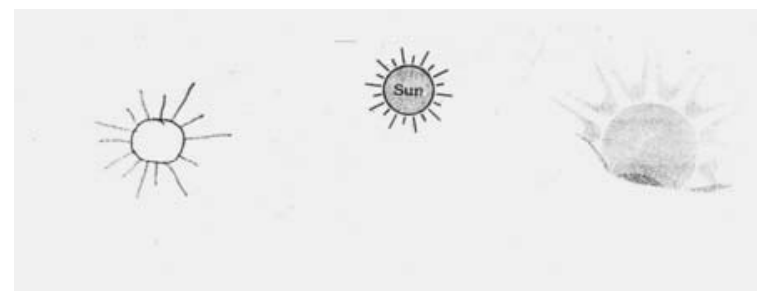

Figure 3. Three different ways of depicting sun: taken from the module 'Images in optics'

What understanding do these indicate about the way light travels? Think about the following:

Rays are physical objects that can be seen.

Light is visible from anywhere 
Only one ray emerges from a point on the light source.

A light source is drawn below? Can you suggest a more appropriate way of drawing it?( a candle was drawn).

This query elicited different kinds of responses. Two are shown below:

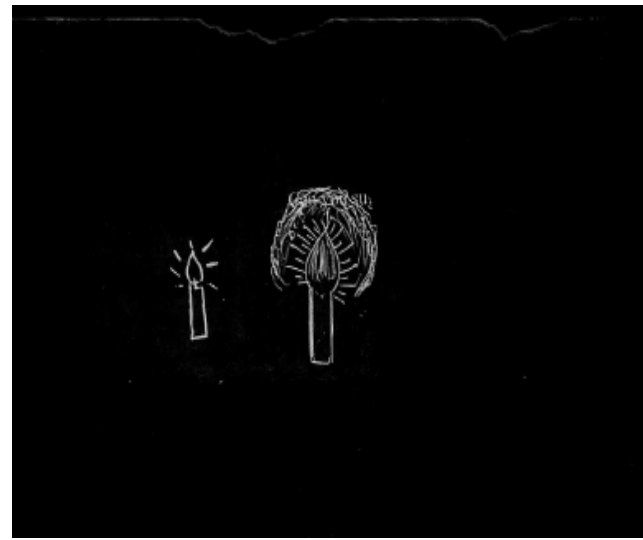

Figure 4. A response categorized as being negatively indicative

This picture drawn by a participant has been categorized as being negatively indicative as the basic idea that only one ray emanates from a point has been retained.

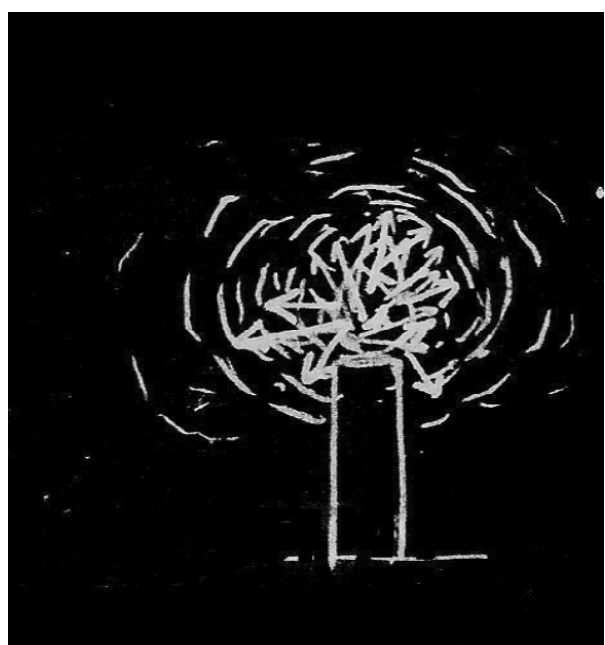

Figure 5. A response categorized as being positively indicative

This diagram has been categorized as being positively indicative as many rays are seen emanating from a point and spreading out.

Beginning with this kind of image analysis the future teachers were then asked to trace the path of the light rays as they traveled from the source to a mirror or a lens. They were asked to predict the nature, location and orientation of the images in the light of the path traveled by the light beam.

For each module, a response table was prepared. This table depicted alongside each query posed in the module, the key features of the responses, categorization of these responses and the codes of students who gave these responses.

\subsection{Post interventional assessment}

A question paralleling the above preinterventional assessment item that was used during post-assessment:

An object $\mathrm{O}$ shaped as shown is placed in front of a plane mirror. Draw the image as and where it will be seen by the observer $\mathrm{A}$ and observer $\mathrm{B}$ ?

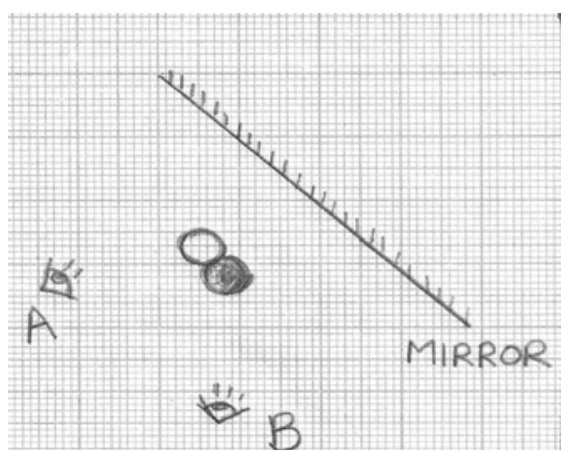

Figure 6. Matched post-interventional assessment item

The features of the image were examined to be able to trace the underlying alternative conceptions. So, if a participant drew two images, it reflected the underlying idea that the image position is determined by the observer position. Similarly other possible alternative ideas could also be examined.

\section{Findings and Discussion}

Of the 27 future teachers who gave an indicative evidence of subscribing to the alternative conception-'In a plane mirror, image distance is determined by observer distance' prior to intervention, 16 gave an indicative evidence of subscribing to the shared conception- 'In a plane mirror, image distance is equal to object distance', post-intervention. Of the 35 future teachers who gave an indicative evidence of subscribing to the alternative conception-'In a plane mirror, image location. Is determined by observer location' prior to intervention, 19 gave an indicative evidence of subscribing to the shared conception-'In a plane mirror, image location is determined by object location post-intervention. Of the 16 future teachers who gave an indicative evidence of subscribing to the alternative conception-'In a plane mirror, rays travel from object location to image location and get laterally inverted' prior to intervention, 11 gave an 
indicative evidence of subscribing to the shared conception 'light rays travel from the object to the mirror, gets reflected and image orientation is decided by the backward extension of the reflected rays' postintervention. The study supports the view that alternative conceptions are tenacious and that teachers or future teachers with advanced academic training may themselves hold many alternative ideas. Further, it is observed that Students even at the level of teacher preparation courses may not always be able to clearly articulate and analyze their own thought processes and need help to be able to do so. Teacher preparation programs therefore need to develop helpful curricular material and create curricular spaces where pre service teachers' beliefs, epistemologies and intuitive notions can be articulated, deliberated upon and challenged.

Though, a majority of future teachers indicated a transformation from the 'alternative' to the 'shared' conception in this case, the results need to be interpreted with caution. The conceptions taken up in this study seem particularly related to understanding of the graphical symbolism in optics but the same may not apply to all the conceptions. The ideas of the participants who chose not to respond to a particular item remain obscure. Further, two students who manifested the shared conception during pre interventional assessment did not respond to the matched post-interventional assessment item and this may be difficult to interpret. It is to be borne in mind that the participants of this study were future teachers with a specified prior engagement with the subject matter and the results may not be easily transferable to a different setting. A change of context will be helpful to corroborate the results. Since the modules were treated as one package, it may be difficult to say which aspect of the modules facilitated this change. For instance an understanding of the role of self, a comprehension of the diagrammatic representation of the path of light travel or an associated feature may all have contributed to eliciting the shared response from a future teacher.

\section{References}

[1] Abimola, I.O. (1996). "Subject Matter Knowledge and Conceptual Change: A Science Education Research Paradigm For Nigeria”, Journal of Science Teaching and Learning, 2(1\&2), pp. 1-7.

[2] Bliss, J. (2008). "Commonsense reasoning about the physical world”, Studies in Science Education, 44(2), pp. 123-155.

[3] Driver, R. (1981). "Pupils' Alternative Frameworks in Science”, European Journal of Science Education, 3(1), pp. 93101 ,

[4] Etkina, E. (2005). "Physics teacher preparation Dreams and Reality”, Journal of Physics Teacher Education Online, 3(2), pp. 3-9.
[5] Galili, I. (1996). "Students' conceptual change in geometrical optics", International Journal of Science Education,18(7), pp. 847-868.

[6] Galili, I. and Hazan, A. (2000). "Learners' knowledge in optics: interpretation,structure and analysis", International Journal of Science Education, 2000, 22(1), pp. 57-88.

[7] Gilbert, J.K. (1983). "Alternative Conceptions: Which Way Now?", Annual Meeting of the American Association of Physics Teachers, New York, Jan 24-27,1983.

[8] Gilbert, J.K. Osborne, R.J. and Fensham, P.J. (1982). "Children's Science and Its Consequences for Teaching”, Science Education, 66(4), pp. 623-633.

[9] Heywood, D.S. (2005). "Primary Trainee Teachers' Learning and Teaching about Light:some pedagogic implications for initial teacher training", International Journal of ScienceEducation, 27(12), pp. 1447-1475.

[10] Lemke, J.L. (1988). "Genres, semantics and classroom education”, Linguistics and Education, 1(1), pp. 81-99.

[11] Mintzes, J.J. and Chiu, M. (2004). ”Understanding and Conceptual Change in Science \& Mathematics: An International Agenda within a Constructivist Framework. Editorial”, International Journal of Science and Mathematics Education, 2(2), pp. 111-114.

[12] Mintzes, J.J.and Wandersee, J.H.( 1998 ). "Research in Science Teaching and Learning: A Human Constructivist View", in Mintzes, J.J., Wandersee, J. H. and Novak, J. D.(Eds.) Teaching Science for Understanding: A Human Constructivist View, California: Academic press.

[13] Mestre J.P. (2001). "Implications of research on learning for the education of prospective science and physics teachers", Physics Education, 36(1), pp. 44-51.

[14] National Council for Teacher Education (2009). National Curriculum Framework For Teacher Education:Towards Preparing Professional and Humane Teacher .New Delhi: NCTE.

[15] Nussbaum, J. (1985). "The Earth as a Cosmic Body”, in Driver,R., Guesne,E. and Tiberghien,A. (Eds.). Children's Ideas in Science, Milton Keynes:Open University Press.

[16] Pardhan, H. and Bano, Y. (2001). "Science teachers' alternate conceptions about direct currents", International Journal of Science Education, 23(3), pp. 301-318.

[17] Reiner, M., Slotta, J.D., Chi, M.T.H. and Resnick, L.B. (2000). "Naïve Physics Reasoning : A Commitment to Substance Based Reasoning”, Cognition and Instruction, 18(1), pp. 1-34.

[18] Waldrip, B., Prain, V. and Carolan, J. (2006). "Learning Junior Secondary Science through Multi- Modal Representations”, Electronic Journal of Science Education, 11(1), pp. 87-107. 